\title{
Diagnostic Usefulness of White Blood Cell and Absolute Neutrophil Count for Postoperative Infection after Anterior Cervical Discectomy and Fusion Using Allograft and Demineralized Bone Matrix
}

\author{
Chae-Gwan Kong, Young-Yul Kim, Chi Young Ahn, Jong-Beom Park \\ Department of Orthopaedic Surgery, Uijeongbu St. Mary's Hospital, The Catholic University of Korea College of Medicine, Uijeongbu, Korea
}

\section{Study Design: Prospective study.}

Purpose: We investigated normative temporal levels of white blood cell (WBC) and absolute neutrophil count (ANC) in uncomplicated anterior cervical discectomy and fusion (ACDF) using allograft and demineralized bone matrix (DBM).

Overview of Literature: No study has investigated the diagnostic usefulness of WBC and ANC for postoperative infection following ACDF using allograft and DBM.

Methods: Blood samples of 85 patients, who underwent one or two-level ACDF, were obtained and evaluated before surgery and on the first, third, fifth, seventh, fourteenth, thirtieth, and ninetieth postoperative days. No infection was found in all patients for at least one year follow-up period.

Results: Mean WBC and ANC values increased significantly and reached peak levels on the first postoperative day. The peaked levels rapidly decreased but still remained elevated above the preoperative levels on the third postoperative day. The levels returned close to the preoperative levels on the fifth postoperative day. The mean WBC and ANC values did not get out of their normal reference ranges throughout the follow-up periods. One-level and two-level ACDF exhibited a similar course of postoperative changes in WBC and ANC values and no significant difference in mean levels of WBC and ANC throughout the follow-up periods.

Conclusions: Uncomplicated ACDF using allograft and DBM showed normal values of WBC and ANC during the early postoperative period. Therefore, significant abnormal values of WBC and ANC at an early postoperative period suggest the possibility of the development of acute postoperative infection after ACDF using allograft and DBM.

Keywords: White blood cell; Absolute neutrophil count; Anterior cervical discectomy and fusion; Allograft; Demineralized bone matrix

\section{Introduction}

Anterior cervical discectomy and fusion (ACDF) is an effective surgical procedure for the management of patients with cervical radiculopathy and/or myelopathy secondary to primary cervical spondylosis and/or disc herniation [1-3]. However, various types of ACDF-related complications may occur, which can be troublesome and occasionally catastrophic [4,5]. Among them, acute postoperative infection following $\mathrm{ACDF}$, although very

Received May 12, 2012; Revised May 18, 2012; Accepted May 18, 2012

Corresponding author: Jong-Beom Park

Department of Orthopaedic Surgery, Uijeongbu St. Mary's Hospital, The Catholic University of Korea College of Medicine,

271 Cheonbo-ro, Uijeongbu 480-717, Korea

Tel: +82-31-820-3578, Fax: +82-31-847-3671, E-mail: spinepjb@catholic.ac.kr 
rare, is potentially dangerous and difficult to manage it $[6,7]$. Timely diagnosis and proper management of this potential complication are critical in order to accomplish a good outcome in these patients. However, it is difficult to detect acute postoperative infection early after ACDF because of the vague clinical finding and inconclusive initial radiographic findings.

Autograft is a gold standard graft option for ACDF but has several disadvantages, such as donor site morbidities and limited availability [8-10]. Therefore, allograft with or without a demineralized bone matrix (DBM) have been recently used for ACDF and have achieved satisfactory clinical and radiological outcomes [11,12]. However, allograft and DBM also have several disadvantages, such as risk of disease transmission and immune reaction [13-15]. Moreover, the incidence of postoperative infection after ACDF is reported to be higher in allograft compared to that of autograft $[8,10]$.

White blood cell (WBC) and absolute neutrophil count (ANC) are early-phase inflammatory indexes and have been widely used for the early detection of postoperative wound infection following surgeries [16-20]. However, no study has investigated the diagnostic usefulness of WBC and ANC for postoperative infection after ACDF using allograft and DBM. Knowledge of the normal course of postoperative changes of WBC and ANC values after uncomplicated ACDF using allograft and DBM is a prerequisite to actually use these parameters as effective tools for the early detection of postoperative infection after ACDF using allograft and DBM. The authors therefore performed the current study in order to investigate 1) the normal course of postoperative changes of WBC and ANC values after uncomplicated ACDF using allograft and DBM as a baseline data, 2) to compare results between one-level and two-level ACDF.

\section{Materials and Methods}

A prospective study was performed on 103 consecutive patients who underwent one-level or two-level ACDF using freeze-dried ulna ring allograft (Korea Bone Bank, Seoul, Korea) and DBX (Musculoskeletal Transplant Foundation, Edison, NJ, USA) DBM for cervical radiculopathy and/or myelopathy caused by primary cervical spondylosis and/or disc herniation between January and October 2010. Patients who had previous anterior or posterior cervical spine surgery and a previous history of infection were not included in the current study from the first. Eighteen patients who have at least one abnormal value of $\mathrm{WBC}$ and $\mathrm{ANC}$ preoperatively were excluded from the current study. Consequently, 85 patients were included in the final analyses. There were 40 men and 45 women with an average age of 55.2 years (range, 27-76 years). Fifty-one were one-level ACDF and 34 were twolevel ACDF. There were no differences in demographic data, including gender and age, between one-level and two-level ACDF ( $p>0.05)$, except for operation time (68.1 \pm 14.7 minutes vs. $93.7 \pm 15.2$ minutes, $p<0.001)$ and total blood loss $(42.3 \pm 13.6 \mathrm{~mL}$ vs. $54.9 \pm 17.3 \mathrm{~mL}$, $p<0.001)$. In all patients, we confirmed the absence of infection through clinical evaluations (symptoms, signs, laboratory findings, and radiologic findings) made with a regular follow-up schedule (postoperatively, 2 weeks, 1 month, 3 months, 6 months, 12 months) for at least 1 year.

All ACDFs were performed by a single surgeon using the standard surgical technique, which is the left side approach. DBX DBM was used $0.5 \mathrm{~mL}$ per level. Blood samples were obtained before surgery and on the first, third, fifth, seventh, fourteenth, thirtieth, and ninetieth postoperative days. Blood samples on the fourteenth, thirtieth and ninetieth days were obtained from patients at the outpatient clinic. A prophylactic antibiotic was given preoperatively (immediately before skin incision) and postoperatively for 3 days.

$\mathrm{WBC}$ and ANC were measured using the laser flow cytometry on the XE-2100 (Sysmex, Kobe, Japan) according to the manufacturer's instructions; the normal reference ranges of WBC and ANC were 4,000-10,000/uL and 2,000-7,500/uL, respectively.

We performed a paired $t$-test to investigate the postoperative serial changes of WBC and ANC values at each time to the preoperative level. In addition, we performed the comparisons for the mean values of $\mathrm{WBC}$ and $\mathrm{ANC}$ between one-level and two-level ACDF using Student's $t$ test at each time. Analyses were performed using SPSS for Windows statistical package (ver. 12, SPSS Inc., Chicago, IL, USA) and a $p$-value less than 0.05 was considered significant.

\section{Results}

Fig. 1 demonstrated normal course for postoperative changes regarding mean $\mathrm{WBC}$ values in uncomplicated 
ACDF using allograft and DBX DBM throughout the follow-up period. The mean WBC value significantly increased and reached peak levels on the first postoperative day compared to the preoperative level. The peaked level gradually decreased but still remained elevated above the preoperative level on the third postoperative day. It returned close to the preoperative level on the fifth postoperative day. However, the mean WBC values did not go beyond the normal reference ranges throughout the follow-up periods. One-level and two-level ACDF ex-

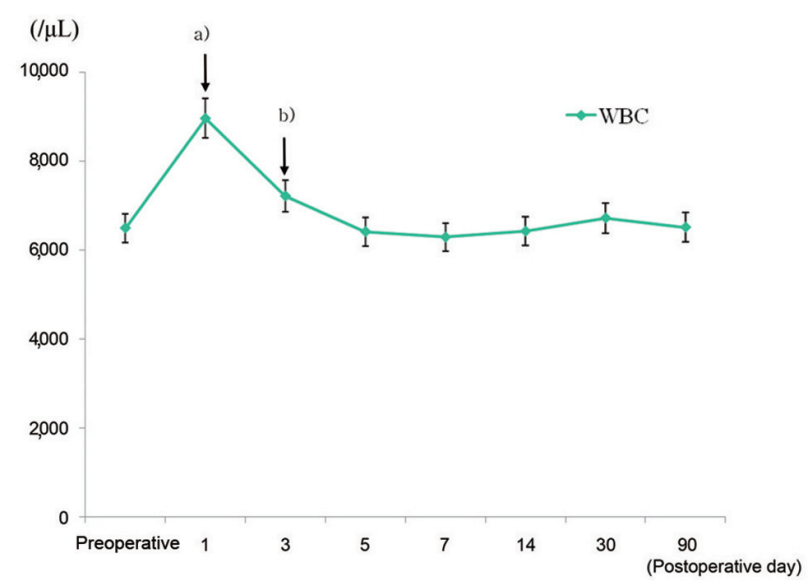

Fig. 1. Normal course of postoperative changes for mean white blood cell (WBC) values in uncomplicated anterior cervical discectomy and fusion using allograft and demineralized bone matrix. ${ }^{\text {a) Indicates }}$

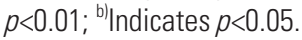

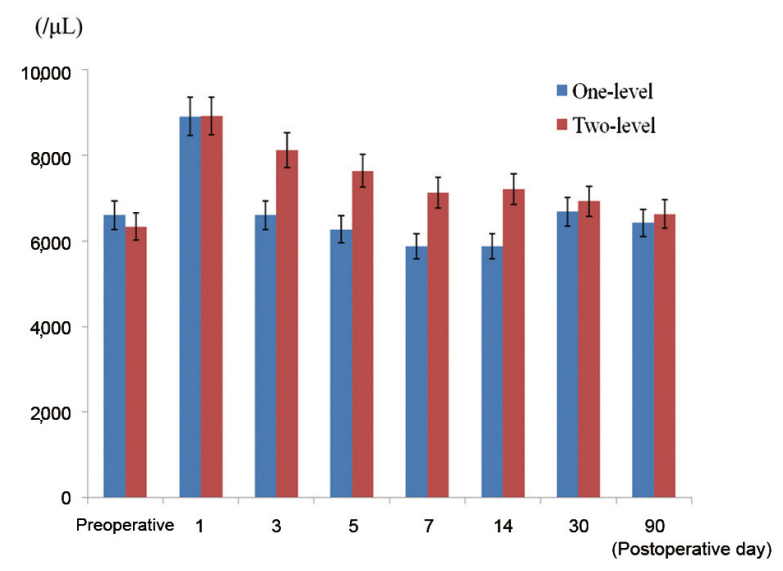

Fig. 2. One-level and two-level anterior cervical discectomy and fusion using allograft and demineralized bone matrix exhibit a similar course of normal postoperative changes for mean white blood cell values throughout the follow-up periods. hibited a similar normal course of postoperative changes regarding mean $\mathrm{WBC}$ values and no significant difference in mean WBC values from the first postoperative day to the ninetieth postoperative day (Fig. 2)

Fig. 3 demonstrated a normal course of postoperative changes for mean ANC values in uncomplicated ACDF using allograft and DBX DBM throughout the follow-up period. The mean ANC value significantly increased and reached peak levels on the first postoperative day compared to the preoperative level. The peaked level gradu-

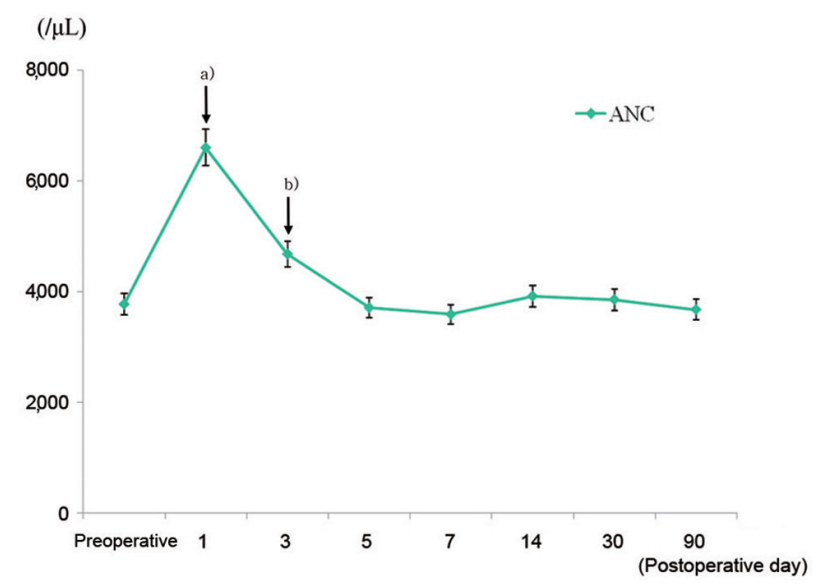

Fig. 3. Normal course of postoperative changes of mean absolute neutrophil count (ANC) values in uncomplicated anterior cervical discectomy and fusion using allograft and demineralized bone matrix.

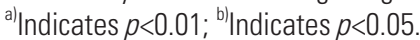

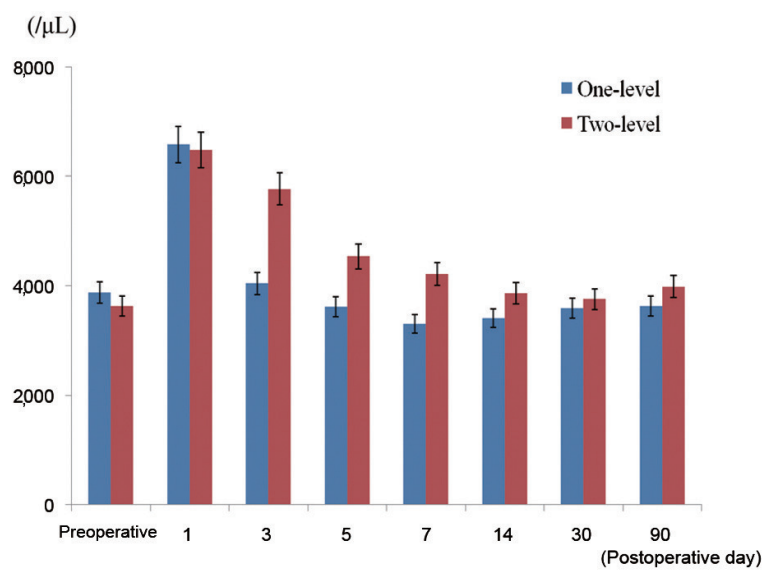

Fig. 4. One-level and two-level anterior cervical discectomy and fusion using allograft and demineralized bone matrix exhibit a similar course of normal postoperative changes for mean absolute neutrophil count values throughout the follow-up periods. 
ally decreased but still remained elevated above the preoperative level on the third postoperative day. It returned close to the preoperative level on the fifth postoperative day. However, the mean ANC values did not go beyond the normal reference ranges throughout the followup periods. One-level and two-level ACDF exhibited a similar course of normal postoperative changes for mean $\mathrm{WBC}$ values and no significant difference in mean ANC values from the first postoperative day to the ninetieth postoperative day (Fig. 4)

\section{Discussion}

The current study regarding uncomplicated ACDF using allograft and DBM for primary cervical spondylosis and/ or disc herniation demonstrated that mean WBC and ANC values increased rapidly and reached peak levels on the first postoperative day, respectively. After the peak, mean WNC and ANC levels rapidly decreased but still remained elevated above the preoperative level on the third postoperative day. Also, mean WNC and ANC levels returned close to preoperative level on the fifth postoperative day. The mean WBC and ANC values did not go beyond their normal reference ranges throughout the follow-up periods. In addition, one-level and two-level ACDF exhibited a similar course of normal postoperative changes in WBC and ANC values and no significant difference in mean levels of WBC and ANC throughout the follow-up periods.

Postoperative infection after ACDF usually occurs within the early postoperative period, especially within the first few days and weeks $[4,5]$. Postoperative infection after ACDF poses difficult management problems, such as extensive long-term antibiotic therapy, reoperations, and prolonged hospital stay. Early and accurate diagnosis is a crucial factor for the successful management of postoperative infection following ACDF. However, it is difficult to accurately detect acute postoperative infection after ACDF before the seventh postoperative day because clinical findings are vague and initial radiographic findings are inconclusive. Therefore, the simple and reliable screening of blood tests for WBC and ANC has been used in order to diagnose acute postoperative infection after spine surgery early [16-20].

According to previous studies, the incidence of postoperative infection after ACDF varies between 0.1\% and $3 \%$ and is higher in allograft compared to autograft
$[6,7]$. Whereas, autograft offers complete histocompatibility and virtually no inflammatory encapsulation of the graft material, various kinds of allograft will produce a spectrum of immunogenic responses from the host. The intensity of the immune response, which occurs, depends on the degree of enzymatic degradation of the native allograft protein. Human allograft is available in two forms: mineralized and demineralized [13-15]. Among the mineralized allograft, freeze-dried allograft is processed by removing water through a vacuum process and is less immunogenic. DBM is a type of allograft bone processed by decalcification of the cortical bone. During the demineralization process, all cellular elements as well as acidsoluble proteins are removed in order to eliminate immunogenicity. Therefore, DBM is weakly immunogenic.

In the current study, one-level and two-level ACDF using allograft and DBM exhibited a similar course of normal postoperative changes regarding $\mathrm{WBC}$ and $\mathrm{ANC}$ values throughout the follow-up period. There was no significant difference in the mean levels of WBC and ANC between one-level and two-level ACDF using allograft and DBM. These findings suggest that the dose of DBM $(0.5 \mathrm{~mL}$ and $1 \mathrm{~mL})$ and the number of allograft (one and two) used in one-level and two-level ACDF makes no difference in immune responses due to the weakened immunogenicity of freeze-fried allografts and DBMs. Although uncomplicated ACDF using allograft and DBM significantly increased normative temporal values of WBC and ANC after surgery, the mean WBC and ANC values did not go beyond their normal reference ranges throughout the follow-up periods. Therefore, the authors cannot say that the use of allograft and DBM further enhances immune responses compared to autograft since we did not have ACDF using autograft in the current study as control. Further study is needed in order to investigate this issue.

\section{Conclusions}

The current study demonstrates that uncomplicated ACDF using allograft and DBM showed normal values of $\mathrm{WBC}$ and $\mathrm{ANC}$ during the early postoperative period. Therefore, significant abnormal values of WBC and ANC at some point during the early postoperative period suggest the development of acute postoperative infection following ACDF using allograft and DBM. 


\section{Conflict of Interest}

No potential conflict of interest relevant to this article was reported.

\section{References}

1. Albert TJ, Murrell SE. Surgical management of cervical radiculopathy. J Am Acad Orthop Surg 1999;7:368-76.

2. Rao RD, Currier BL, Albert TJ, et al. Degenerative cervical spondylosis: clinical syndromes, pathogenesis, and management. J Bone Joint Surg Am 2007;89:1360-78.

3. Martin GJ Jr, Haid RW Jr, MacMillan M, Rodts GE Jr, Berkman R. Anterior cervical discectomy with freeze-dried fibula allograft: overview of 317 cases and literature review. Spine (Phila Pa 1976) 1999;24:852-8.

4. Fountas KN, Kapsalaki EZ, Nikolakakos LG, et al. Anterior cervical discectomy and fusion associated complications. Spine (Phila Pa 1976) 2007;32:2310-7.

5. Park JB, Cho YS, Riew KD. Development of adjacentlevel ossification in patients with an anterior cervical plate. J Bone Joint Surg Am 2005;87:558-63.

6. Bassewitz HL, Fischgrund JS, Herkowitz HN. Postoperative spine infections. Semin Spine Surg 2000;12:203-11.

7. Sasso RC, Garrido BJ. Postoperative spinal wound infections. J Am Acad Orthop Surg 2008;16:330-7.

8. Miller LE, Block JE. Safety and effectiveness of bone allografts in anterior cervical discectomy and fusion surgery. Spine (Phila Pa 1976) 2011;36:2045-50.

9. Arrington ED, Smith WJ, Chambers HG, Bucknell AL, Davino NA. Complications of iliac crest bone graft harvesting. Clin Orthop Relat Res 1996;(329):300-9.

10. Schnee CL, Freese A, Weil RJ, Marcotte PJ. Analysis of harvest morbidity and radiographic outcome using autograft for anterior cervical fusion. Spine (Phila
Pa 1976) 1997;22:2222-7.

11. Deutsch H, Haid R, Rodts G Jr, Mummaneni PV. The decision-making process: allograft versus autograft. Neurosurgery 2007;60:S98-102.

12. Floyd T, Ohnmeiss D. A meta-analysis of autograft versus allograft in anterior cervical fusion. Eur Spine J 2000;9:398-403.

13. An HS, Simpson JM, Glover JM, Stephany J. Comparison between allograft plus demineralized bone matrix versus autograft in anterior cervical fusion. A prospective multicenter study. Spine (Phila Pa 1976) 1995;20:2211-6.

14. Dinopoulos HT, Giannoudis PV. Safety and efficacy of use of demineralised bone matrix in orthopaedic and trauma surgery. Expert Opin Drug Saf 2006;5:847-66.

15. Khan SN, Fraser JF, Sandhu HS, Cammisa FP Jr, Girardi FP, Lane JM. Use of osteopromotive growth factors, demineralized bone matrix, and ceramics to enhance spinal fusion. J Am Acad Orthop Surg 2005;13:129-37.

16. Carr WP. The role of the laboratory in rheumatology. Acute-phase proteins. Clin Rheum Dis 1983;9:22739.

17. Palosuo T, Husman T, Koistinen J, Aho K. C-reactive protein in population samples. Acta Med Scand 1986;220:175-9.

18. Takahashi J, Ebara S, Kamimura M, et al. Earlyphase enhanced inflammatory reaction after spinal instrumentation surgery. Spine (Phila Pa 1976) 2001;26:1698-704.

19. Mok JM, Pekmezci M, Piper SL, et al. Use of C-reactive protein after spinal surgery: comparison with erythrocyte sedimentation rate as predictor of early postoperative infectious complications. Spine (Phila Pa 1976) 2008;33:415-21.

20. Meyer B, Schaller K, Rohde V, Hassler W. The Creactive protein for detection of early infections after lumbar microdiscectomy. Acta Neurochir (Wien) 1995;136:145-50. 